This item was submitted to Loughborough's Research Repository by the author.

Items in Figshare are protected by copyright, with all rights reserved, unless otherwise indicated.

\title{
Transient analysis of isothermal elastohydrodynamic circular point contacts
}

PLEASE CITE THE PUBLISHED VERSION

PUBLISHER

Professional Engineering Publishing / @ IMECHE

VERSION

VoR (Version of Record)

LICENCE

CC BY-NC-ND 4.0

REPOSITORY RECORD

Jalali-Vahid, D., Homer Rahnejat, Z.M. Jin, and D. Dowson. 2019. "Transient Analysis of Isothermal Elastohydrodynamic Circular Point Contacts". figshare. https://hdl.handle.net/2134/4775. 
This item was submitted to Loughborough's Institutional Repository (https://dspace.lboro.ac.uk/) by the author and is made available under the following Creative Commons Licence conditions.

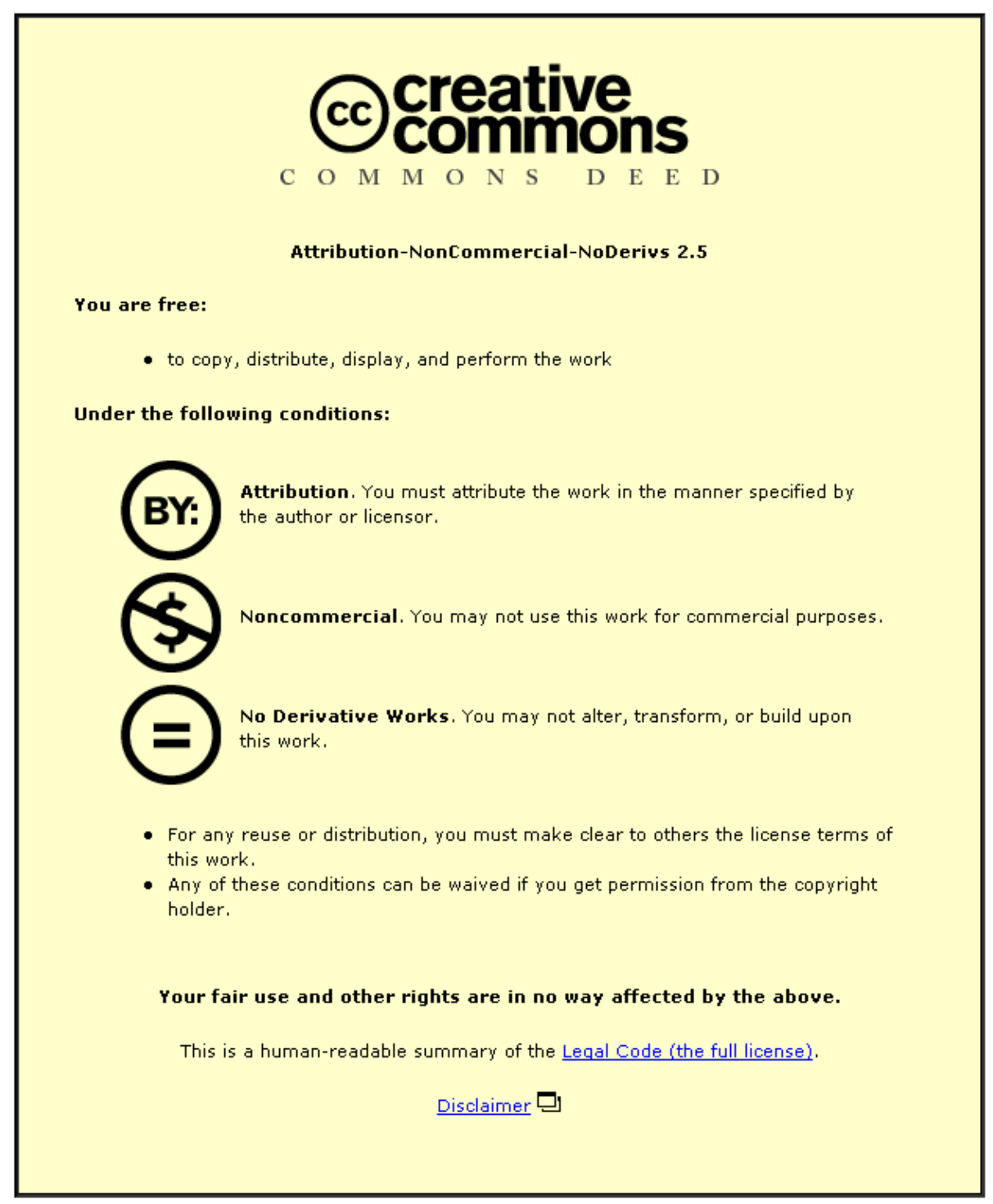

For the full text of this licence, please go to: http://creativecommons.org/licenses/by-nc-nd/2.5/ 


\title{
Transient analysis of isothermal elastohydrodynamic circular point contacts
}

\author{
D Jalali-Vahid ${ }^{1}$, H Rahnejat ${ }^{2}, \mathbf{Z}$ M Jin ${ }^{1}$ and D Dowson ${ }^{2 *}$ \\ ${ }^{1}$ Department of Mechanical Engineering, University of Bradford, UK \\ ${ }^{2}$ Department of Mechanical Engineering, Loughborough University, UK
}

\begin{abstract}
In this paper a solution method is presented for the transient isothermal elastohydrodynamic lubrication of point contact conjunctions, based upon the Newton-Raphson scheme and low iteration relaxation. The numerical results are compared with the numerical and experimental observations of others for the circular point contact of a ball against a flat glass disc under oscillating conditions. Good agreement has been found with other numerical solutions. The comparison with the experimental results shows good qualitative agreement.
\end{abstract}

Keywords: circular point contacts, transient isothermal elastohydrodynamic lubrication

\section{NOTATION}

a semi-major axis of the contact ellipse in the transverse direction $(\mathrm{m})$

$b \quad$ semi-minor axis of the contact ellipse in the entraining direction $(\mathrm{m})$

$D$ deformation matrix (m)

$E \quad$ modulus of elasticity $(\mathrm{Pa})$

$E^{\prime} \quad$ equivalent Young's modulus $=2 /\left[\left(1-v_{\mathrm{A}}^{2}\right) / E_{\mathrm{A}}+\left(1-v_{\mathrm{B}}^{2}\right) / E_{\mathrm{B}}\right](\mathrm{Pa})$

$G^{*} \quad$ material parameter $=\alpha E^{\prime}$

$H_{i, j}(\bar{t})$ dimensionless film thickness as a function of time $=h_{i, j}(t) R_{x} / b^{2}$

$H_{0}(\bar{t}) \quad$ dimensionless constant defined in equation (4)

$K \quad$ ellipticity parameter $=a / b$

$M \quad$ number of nodes in the $x$ direction

$n \quad$ time step

$N \quad$ number of nodes in the $y$ direction

$P \quad$ dimensionless pressure $=p / P_{\mathrm{h}}$

$P_{\mathrm{h}} \quad$ maximum Hertzian pressure

$R_{x} \quad$ equivalent radius of contact in the $x$ direction $(\mathrm{m})$

$S_{i, j} \quad$ shape of the contacting surface $h \quad$ film thickness (m)

$p \quad$ pressure $(\mathrm{Pa})$

time (s)

dimensionless time $=\left(t u_{\mathrm{av}}\right) / b$

$T \quad$ period (s)

$u_{\mathrm{e}} \quad$ mean velocity components in the $x$ direction $=\left(u_{1}+u_{2}\right) / 2(\mathrm{~m} / \mathrm{s})$

$u_{0} \quad$ nominal velocity $(\mathrm{m} / \mathrm{s})$

$u_{1} \quad$ surface velocity of the sphere in the $x$ direction $(\mathrm{m} / \mathrm{s})$

$u_{2} \quad$ surface velocity of the plane in the $x$ direction $(\mathrm{m} / \mathrm{s})$

$U^{*} \quad$ dimensionless velocity $=\left(u \eta_{0}\right) /\left(E^{\prime} R_{x}\right)$

$W \quad$ normal applied load $(\mathrm{N})$

$W_{0} \quad$ nominal load $(\mathrm{N})$

$W^{*} \quad$ dimensionless load $=W /\left(E^{\prime} R^{2}\right)$

$x \quad$ Cartesian coordinate

$X \quad x / b$

$y \quad$ Cartesian coordinate

$Y \quad y / a$

$z \quad$ viscosity pressure index

$\alpha \quad$ pressure-viscosity coefficient $\left(\mathrm{m}^{2} / \mathrm{N}\right)$

$\delta_{i, j}(\bar{t})$ total elastic deformation as a function of time $\Delta t \quad$ time interval (s).

$\Delta X$ distance between two neighbouring points in the $X$ direction

$\Delta Y \quad$ distance between two neighbouring points in the $Y$ direction

$\eta \quad$ lubricant viscosity (Pa s)

The MS was received on 21 January 1999 and was accepted after revision for publication on 25 April 2001.

*Corresponding author: Ryedale, 23 Church Lane, Adel, Leeds LS16 $8 D Q, U K$. $\bar{\eta} \quad$ dimensionless lubricant viscosity $=\eta / \eta_{0}$

$\eta_{0} \quad$ atmospheric viscosity ( $\mathrm{Pa} \mathrm{s}$ )

$\theta \quad$ angle $=\omega t$ 
$v \quad$ Poisson's ratio

$\rho \quad$ lubricant density $\left(\mathrm{kg} / \mathrm{m}^{3}\right)$

$\rho_{0} \quad$ atmospheric density $\left(\mathrm{kg} / \mathrm{m}^{3}\right)$

$\bar{\rho} \quad$ dimensionless density $=\rho / \rho_{0}$

$\omega \quad$ angular velocity

$\Omega \quad$ under-relaxation factor

\section{INTRODUCTION}

A considerable amount of research has been devoted to the study of elastohydrodynamic lubrication (EHL) in machine elements such as bearings, gears and cam and follower pairs. Most of the initial investigations were concerned with the study of lubricant film thickness under steady state conditions. The absence of wear under conditions in which steady state analysis predicted zero or exceedingly small film thickness remained a significant obstacle. These included the diminution of the lubricant film on the sharp rise and fall of cam lift in the vicinity of the cam nose, where inlet boundary reversals take place. Although the contact transit time is short lived and the lubricant film is rapidly replenished, the predicted steady state oil-film thickness was insufficient to guard against wear. The same problem was also noted in bearings and gears in cold start-up conditions or at low speeds of entraining motion. A larger class of problem was also discovered, where the presence of a lubricant film under large impact loads (in, for example, crankshaft journal bearings) or with no entraining velocity (as in synovial joints in stationary positions) could not be explained. The search for an explanation led to the recognition of the significance of squeeze-film action, providing enhanced load-carrying capacity under transient conditions.

Initial solutions for pure squeeze action were obtained as early as the 1950s by Christensen [1] for hydrodynamic conditions with isoviscous and piezoviscous lubricants. Similar studies were also undertaken by Herrebrugh [2]. Later, Christensen [3], Conway [4] and Lee and Cheng [5] reported pure squeeze solutions under elastohydrodynamic conditions. More recent solutions have been reported for both rigid and elastic ellipsoids by Bedewi et al. [6] and Rahnejat [7, 8]. The latter also reported solutions for combined entraining and squeeze-film action under quasi-static conditions for finite line contacts of roller bearings. A similar solution for elliptical point contact conditions has been reported by Mostofi and Gohar [9]. A mixed regime of lubrication, including an extrapolated equation with the effect of squeeze-film motion from reference [9], was included in the model of a deep groove ball bearing under dynamic conditions by Rahnejat and Gohar [10]. However, in all these analyses the effect of inertial dynamics was ignored.

Transient solutions, particularly for isothermal elastohydrodynamic conditions, have recently been obtained by a number of researchers. These include the normal bouncing of an elastic ball on an oily plate by Dowson and Wang [11]. An interesting feature of the transient response was observed to be the rather sharp and very large rise in pressure during the rebound of the ball. These findings conformed well with the experimental observations of Safa and Gohar [12]. These transient solutions pave the way for realistic investigations of the dynamics of ball and rolling bearing contacts under cyclic loading, when the rolling element enters or leaves the loaded or unloaded regions of the bearing that are created by the mutual convergence or separation of bearing rings. Nevertheless, a complete solution for the entire bearing remains computationally elusive and calls for very large memory requirements and exceedingly long computation times. In this case the only practicable option still remaining is the quasi-static solution for the lubricant film thickness for a particular film location and in very small steps of time, as reported in references [7], [8] and [10]. The drawback is that the squeeze velocity employed can only be considered as an average value, as opposed to an instantaneous one determined by the prevailing transient conditions.

This problem can be overcome by means of a combined solution of inertial dynamics and transient EHL for a single ball (representing typical conditions during the orbital traverse under steady state bearing oscillations, i.e. limit cycle vibrations). Such an approach has been highlighted by Wijnant and Venner [13], who have shown that with dominating entraining motion the transient solutions approach the equivalent steady state solutions. When a significant squeeze-film action takes place, the instantaneous value of the squeeze-film velocity diverges from its steady state average value; this leads to noticeable differences between the transient and quasi-static solutions. The observations of Wijnant and Venner were also in line with those of Dowson and Wang [11] and Safa and Gohar [12].

A numerical algorithm that combines the NewtonRaphson scheme and low relaxation iteration for the solution of general entraining and squeeze-film action in point contact EHL problems is presented in this paper. This method has also been employed by Ai and $\mathrm{Yu}$ [14] for the case of line contact transient EHL problems. Furthermore, this paper provides solutions that conform well with the optical interferometric studies under oscillating conditions reported by Ren et al. [15]. Even better agreement is obtained with the numerical results of $\mathrm{Oh}[\mathbf{1 6}]$.

\section{BACKGROUND THEORY}

\subsection{Reynolds equation}

The general form of the Reynolds equation for transient 
conditions is given as

$$
\begin{aligned}
& \frac{\partial}{\partial x}\left(\frac{\rho h^{3}}{\eta} \frac{\partial p}{\partial x}\right)+\frac{\partial}{\partial y}\left(\frac{\rho h^{3}}{\eta} \frac{\partial p}{\partial y}\right) \\
& \quad=12\left[u_{\mathrm{av}} \frac{\partial(\rho h)}{\partial x}+\rho \frac{\partial h}{\partial t}+h \frac{\partial \rho}{\partial t}\right]
\end{aligned}
$$

where the following dimensionless groups apply:

$$
\begin{array}{llll}
X=\frac{x}{b}, & \bar{\rho}=\frac{\rho}{\rho_{0}}, & H=\frac{h R_{x}}{b^{2}}, & P=\frac{p}{P_{\mathrm{h}}} \\
Y=\frac{y}{a}, & \bar{\eta}=\frac{\eta}{\eta_{0}}, & \bar{t}=\frac{u_{\mathrm{av}} t}{b}, & U^{*}=\frac{u \eta_{0}}{R_{x} E^{\prime}}
\end{array}
$$

Thus

$$
\begin{gathered}
\frac{\partial}{\partial X}\left(\frac{\bar{\rho} H^{3}}{\bar{\eta}} \frac{\partial P}{\partial X}\right)+\frac{1}{K^{2}} \frac{\partial}{\partial Y}\left(\frac{\bar{\rho} H^{3}}{\bar{\eta}} \frac{\partial P}{\partial Y}\right) \\
-\lambda\left[\frac{\partial(\bar{\rho} H)}{\partial X}-\left(\bar{\rho} \frac{\partial H}{\partial \bar{t}}+H \frac{\partial \bar{\rho}}{\partial \bar{t}}\right)\right]=0
\end{gathered}
$$

where

$$
\lambda=\frac{12 R_{x}^{2} \eta_{0} u_{\mathrm{e}}}{b^{3} P_{\mathrm{h}}}
$$

The last term in equation (3) represents the time dependent portion of the Reynolds equation. It allows for the mutual approach and separation of loaded rolling members. It should be noted that the Reynolds equation is based on the assumption that the inertial forces within the fluid are small compared with the other forces that are present, such as gravity, pressure and shear. This is valid because of the very small mass of fluid that is actually within the contact zone at any given instance.

The boundary conditions that are imposed on the Reynolds equation are taken as

$$
\begin{aligned}
& X=0, \quad X=X_{\text {exit }} \Rightarrow P=0 \\
& Y=0, \quad Y=Y_{\text {exit }} \Rightarrow P=0
\end{aligned}
$$

and for the outlet region

$$
P=\frac{\partial P}{\partial X}=\frac{\partial P}{\partial Y}=0
$$

\subsection{Elastic film shape}

The film thickness between the contacting surfaces is described as

$$
H_{i, j}(\bar{t})=H_{0}(\bar{t})+S_{i, j}+\delta_{i, j}(\bar{t})
$$

where $\delta_{i j}(\bar{t})$ is the total elastic deformation of the contiguous bodies in contact. In non-dimensional form, $\delta_{i, j}(\bar{t})$ can be written as

$$
\delta_{K, L}=\frac{2}{\pi} \frac{P_{\mathrm{h}}}{E^{\prime}} \sum_{j=1,2, \ldots}^{N} \sum_{i=1,2, \ldots}^{M} P_{i, j}(\bar{t}) D_{i^{*}, j^{*}}
$$

where according to Johnson [17]

$$
\begin{aligned}
& i^{*}=|K-i|+1 \\
& j^{*}=|L-j|+1
\end{aligned}
$$

The contact influence coefficient matrix, $D$, is provided in Appendix 1.

\subsection{Density-pressure relationship}

The lubricant density variation with pressure is defined by Dowson and Higginson [18] as

$$
\bar{\rho}(P)=1+\frac{\varepsilon P_{\mathrm{h}} P}{1+\zeta P_{\mathrm{h}} P}
$$

where $\varepsilon$ and $\zeta$ are constants related to the type of lubricant employed.

\subsection{Viscosity-pressure relationship}

The variation in lubricant viscosity with pressure is given by Roelands [19] as

$$
\bar{\eta}=\left\{\frac{\eta_{\infty}}{\eta_{0}}\right\}^{\left[1-\left(1+P_{\mathrm{h}} P / \gamma\right)^{z}\right]}
$$

where $z$ is the viscosity pressure index, $\eta_{\infty}=$ $0.631 \times 10^{-4} \mathrm{~Pa} \mathrm{~s}$ and $\gamma=1.9609 \times 10^{8} \mathrm{~N} / \mathrm{m}^{2}$.

\subsection{Load equation}

At any instant of time, the external load is balanced by integrated oil-film pressure distribution. In nondimensional form, the instantaneous load equation can be written as

$$
\int_{-\infty}^{\infty} \int_{-\infty}^{\infty} P(X, Y) \mathrm{d} X \mathrm{~d} Y=\frac{2}{3} \pi
$$

\section{NUMERICAL SOLUTION}

By using a mixed second-order central and first-order backward difference in space and backward difference 
in time, the dimensionless Reynolds' equation at each location $i, j$ can be written as

$$
\begin{aligned}
& F_{i, j}=\frac{1}{2 \Delta X^{2}}\left[\left(\frac{\bar{\rho} H^{3}}{\bar{\eta}}\right)_{i+1, j}^{n}+\left(\frac{\bar{\rho} H^{3}}{\bar{\eta}}\right)_{i, j}^{n}\right] P_{i+1, j}^{n} \\
& +\frac{1}{2 \Delta X^{2}}\left[\left(\frac{\bar{\rho} H^{3}}{\bar{\eta}}\right)_{i, j}^{n}+\left(\frac{\bar{\rho} H^{3}}{\bar{\eta}}\right)_{i-1, j}^{n}\right] P_{i-1, j}^{n} \\
& +\frac{1}{2 K^{2} \Delta Y^{2}}\left[\left(\frac{\bar{\rho} H^{3}}{\bar{\eta}}\right)_{i, j+1}^{n}+\left(\frac{\bar{\rho} H^{3}}{\bar{\eta}}\right)_{i, j}^{n}\right] P_{i, j+1}^{n} \\
& +\frac{1}{2 K^{2} \Delta Y^{2}}\left[\left(\frac{\bar{\rho} H^{3}}{\bar{\eta}}\right)_{i, j}^{n}+\left(\frac{\bar{\rho} H^{3}}{\bar{\eta}}\right)_{i, j-1}^{n}\right] P_{i, j-1}^{n} \\
& -\frac{1}{2 \Delta X^{2}}\left[\left(\frac{\bar{\rho} H^{3}}{\bar{\eta}}\right)_{i+1, j}^{n}+2\left(\frac{\bar{\rho} H^{3}}{\bar{\eta}}\right)_{i, j}^{n}\right. \\
& \left.+\left(\frac{\bar{\rho} H^{3}}{\bar{\eta}}\right)_{i-1, j}^{n}\right] P_{i, j}^{n} \\
& -\frac{1}{2 K^{2} \Delta Y^{2}}\left[\left(\frac{\bar{\rho} H^{3}}{\bar{\eta}}\right)_{i, j+1}^{n}+2\left(\frac{\bar{\rho} H^{3}}{\bar{\eta}}\right)_{i, j}^{n}\right. \\
& \left.+\left(\frac{\bar{\rho} H^{3}}{\bar{\eta}}\right)_{i, j-1}^{n}\right] P_{i, j}^{n} \\
& -\lambda\left(\frac{(\bar{\rho} H)_{i+1, j}-(\bar{\rho} H)_{i-1, j}}{2 \Delta X}\right)^{n} \\
& -\lambda\left\{\rho _ { i , j } ^ { n } \left(\frac{H_{i, j}^{n}-H_{i, j}^{n-1}}{\Delta \bar{t}}+\frac{2}{\pi} \frac{P_{\mathrm{h}}}{E^{\prime}}\right.\right. \\
& \left.\times \sum_{L=1,2, \ldots}^{N} \sum_{K=1,2, \ldots}^{M} D_{i^{*}, j^{*}} \frac{P_{k, l}^{n}-P_{k, l}^{n-1}}{\Delta \bar{t}}\right) \\
& \left.+H_{i, j}^{n}\left[\frac{\varepsilon}{1+\zeta P_{i, j}^{n}}\left(1-\frac{\zeta P_{i, j}^{n}}{1+\zeta P_{i, j}^{n}}\right) \frac{P_{i, j}^{n}-P_{i, j}^{n-1}}{\Delta \bar{t}}\right]\right\}
\end{aligned}
$$

The complete discretized forms for all the partial derivatives are given in Appendix 2.

Since the pressure profile and the film thickness must be compatible, the coupled Reynolds, elasticity and load balance equations must be solved simultaneously in the contact conjunction at each time step $n$. In this study the Newton-Raphson method is used to solve the coupled system equation. Therefore, the modified Newton-Raphson equation for the Reynolds' equation (3) can be expressed as

$$
\sum_{l=2}^{M-1} \sum_{k=2}^{N-1} \mathbf{J}_{i j, k l} \Delta P_{k, l}=-F_{i, j}
$$

for

$$
2 \leqslant i \leqslant M-1 \quad \text { and } \quad 2 \leqslant j \leqslant N-1
$$

The Jacobian matrix is given by the following relation:

$$
\mathbf{J}_{i j, k l}=\frac{\partial F_{i, j}}{\partial P_{k, l}}
$$

To save in computer storage, the algorithm of Hsu and Lee [20] is extended for transient EHL problems where $\partial F_{i, j} / \partial F_{k, l}$ are set to zero for $k$ less than $i-1$ and greater than $i+1$, and for $l$ less than $j-1$ and greater than $j+1$. Therefore, the Jacobian matrix decreases from nine terms in each row to five terms. In order to avoid computer storage and computing time problems for the matrix inversion in the Gauss elimination method, the GaussSeidel iterative method is used to solve the system equations (10). Therefore, the system equations can be rewritten as

$$
\Delta P_{k, l}^{n}=\frac{\begin{array}{c}
-F_{k, l}-J_{k l, k-1 l} \Delta P_{k-1 l}^{n}-J_{k l, k+1 l} \Delta P_{k+1 l}^{n-1} \\
-J_{k l, k l-1} \Delta P_{k l-1}^{n}-J_{k l, k l+1} \Delta P_{k l+1}^{n-1}
\end{array}}{J_{k l, k l}}
$$

The pressure can be updated by using an underrelaxation factor, $\Omega$, as follows:

$$
P_{i, j}^{\text {new }}=P_{i, j}^{\text {old }}+\Omega \Delta P_{i, j}
$$

The numerical algorithm starts from a steady state solution, when $W=W_{0}$. The cyclic loading period, $t_{\mathrm{c}}=$ $2 \pi / \omega$, is divided into 512 steps. In the second time step, by changing the applied load and using $P_{i, j}$ and $H_{i, j}$ from the previous time steps, the transient Reynolds equation is solved and the pressure distribution for the current time step is obtained. The convergence of pressure is checked and, if the convergence criterion is satisfied, the total force is computed. The last step is to check the force balance equation for this time step. If it is satisfied, the programme will go to the next time step or $H_{0}(\bar{t})$ is changed and the transient Reynolds equation is solved to determine the new pressure distribution. The programme is continued until a periodic solution is achieved. In general, a periodic solution can be achieved within five cycles.

Finally, the convergence criteria for the pressure and load balance equations are

$$
\begin{aligned}
& \left(\frac{\sum_{i} \sum_{j}\left(P_{i, j}^{\text {new }}-P_{i, j}^{\text {old }}\right)^{2}}{M \times N}\right)^{1 / 2} \leqslant 10^{-4} \\
& \left|\iint P(X, Y) \mathrm{d} X \mathrm{~d} Y-\frac{2}{3} \pi\right| \leqslant 10^{-4}
\end{aligned}
$$




\section{SIMULATION RESULTS}

Nishikawa et al. [21] have studied the transient EHL problem in the contact of a steel ball and a reciprocating glass disc under both pure rolling or pure sliding. The diameter of the ball was $25.4 \mathrm{~mm}$ and the reduced elastic modulus for the contact was $117 \mathrm{GPa}$. Their optical interferometric study revealed that at the centre of the stroke the central oil-film thickness was almost the same as that under unidirectional motion, and that at the end of the stroke the minimum oil-film thickness moved towards the exit side. The test conditions adopted by Nishikawa et al. [21] for the case of pure rolling have been used here in a simulation study. These are: a mineral oil with a kinematic viscosity of $405 \mathrm{~mm}^{2} / \mathrm{s}$ at $40{ }^{\circ} \mathrm{C}$ and $31 \mathrm{~mm}^{2} / \mathrm{s}$ at $100^{\circ} \mathrm{C}$, a specific gravity of 0.884 at $15^{\circ} \mathrm{C}$ and a piezoviscosity index, $\alpha$, of $19 \mathrm{GPa}^{-1}$. The reciprocating sinusoidal motion is represented by the following average entraining velocity, $u_{\mathrm{e}}=\left(u_{1}+u_{2}\right) / 2$, in the contacting region:

$$
u_{\mathrm{e}}=u_{0} \sin (2 \pi t)
$$

Figure 1a shows the experimental results obtained by Nishikawa et al. [21]. The corresponding numerical predictions are shown in Fig. 1b. The numerical results agree well with the experimental findings, in both cases depicting the film shape through the centre of the contact in the direction of entraining motion. They both show that, at the ends of the stroke $(t=0$ and $t=T / 2)$, oil is entrapped between the contiguous bodies. At $t=0$, the entraining motion is towards the right, and the thickness of the local minimum film is greater on the left-hand side (i.e. at the contact inlet ) than at the minimum exit at the right side. This situation is reversed at $t=T / 2$. During the half-cycle (i.e. $0 \leqslant t \leqslant T / 2$ ), the minimum lubricant film thickness is gradually pushed towards the exit as the speed of entraining motion is increased. At $t=T / 4$, there is no rate of change in entraining motion and the film thickness is almost the same as that obtained under steady state unidirectional motion. At the ends of the strokes, the entraining motion ceases and the lubricant film is momentarily sustained by squeeze-film action. The film of oil entrapped between the minima along the centre of the contact is reminiscent of the dimple shape formed under pure squeeze-film motion in, for example, the approach of a ball to an oily plate, observed by Dowson and Wang [11]. At the ends of each stroke, inlet reversal takes place, replenishing the oil-film thickness.

The experimental results presented by Nishikawa et al. [21] cover half the reciprocating cycle. The numerical predictions shown in Fig. 1b represent the full cycle. The results for the second half of the cycle are an almost mirror image of the first half of the cycle. It can be seen that there is good agreement between the numerical predictions and the experimental measurements.

Another simulation run of the model was undertaken to enable a comparison to be made between the theoretical predictions and the experimental results reported by Ren et al. [15], where an optical interferometric study of the EHL film in a nominal point contact under unsteady conditions was carried out. An exciter was used with their ball and plate machine for cyclical variation of the applied load between a steel ball and the optically flat glass plate. The applied cyclic load was given by $W=W_{0}+\Delta W \sin \omega t$. The relevant data are provided in Table 1. The authors provided the film thickness profiles in the direction of entraining motion for the positions of the central and minimum exit. Figure $2 \mathrm{a}$ shows the experimental unsteady oil-film profiles through a cycle of the applied load $(9-69 \mathrm{~N})$. The authors noted that the minimum film thickness along the centre of the contact in the direction of entraining motion remained almost constant with time. Furthermore, they pointed out that in the part of the cycle corresponding to an increasing load (i.e. $-\pi / 2 \sim \pi / 2$ ) the film thickness could increase, while the contact zone expanded. In the subsequent part of the cycle, with a decreasing value of load (i.e. $\pi / 2 \sim 3 \pi / 2$ ), the contact zone decreased in size and the film thickness was reduced. A dimpleshaped film resulted in the outlet region when the load was increasing. This dimple, reminiscent of a pure squeeze effect, is shifted to the outlet region on account

Table 1 Values of parameters used by Ren et al. [15]

\begin{tabular}{llll}
\hline Numbers & Parameters & Symbols & Quantities and formulae \\
\hline 1 & Velocity $(\mathrm{m} / \mathrm{s})$ & $u$ & 0.15 \\
2 & Load $(\mathrm{N})$ & $W$ & $W=W_{0}+30.0 \sin (\omega t)$ \\
3 & Nominal load $(\mathrm{N})$ & $W_{0}$ & 39.0 \\
4 & Angular velocity $(\mathrm{rad} / \mathrm{s})$ & $\omega$ & $2 \pi$ \\
5 & Ball radius $(\mathrm{m})$ & $R$ & 0.0127 \\
6 & Viscosity at $P=0(\mathrm{~Pa} \mathrm{~s})$ & $\eta_{0}$ & 0.6948 \\
7 & Density at $P=0\left(\mathrm{~kg} / \mathrm{cm}^{3}\right)$ & $\rho_{0}$ & $0.85 \times 10^{-3}$ \\
8 & Temperature $\left({ }^{\circ} \mathrm{C}\right)$ & $T$ & 15.0 \\
9 & Poisson's ratio & $v_{1}$ (glass) & 0.25 \\
10 & Poisson's ratio & $\nu_{2}$ (ball, steel) & 0.3 \\
11 & Young's modulus $(\mathrm{Pa})$ & $E_{1}$ (glass) & $0.0746 \times 10^{12}$ \\
12 & Young's modulus $(\mathrm{Pa})$ & $E_{2}$ (ball, steel) & $0.2110 \times 10^{12}$ \\
\hline
\end{tabular}




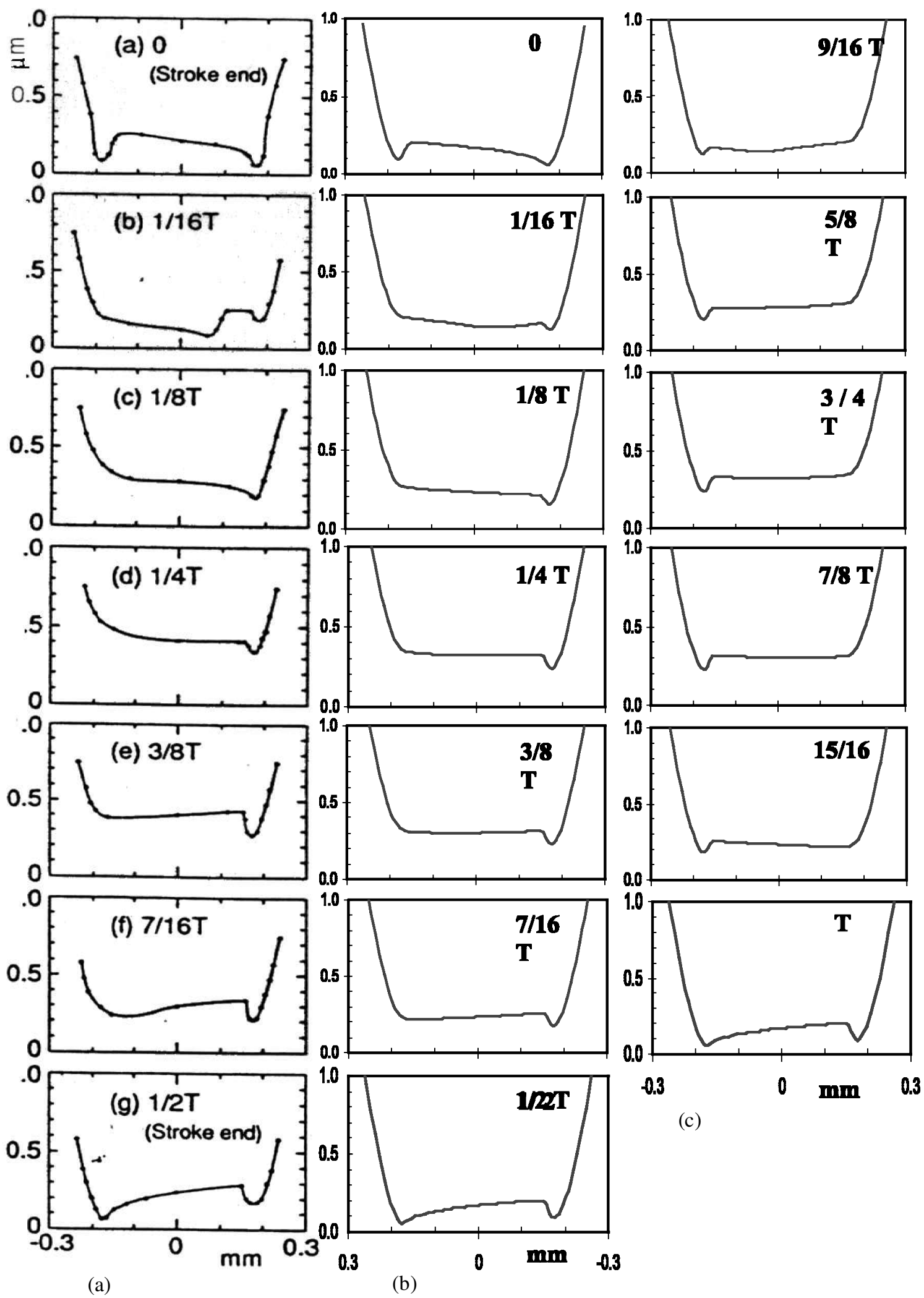

Fig. 1 Transient EHL central film profiles: (a) experimentally measured (after Nishikawa et al. [21]); (b) numerically predicted 

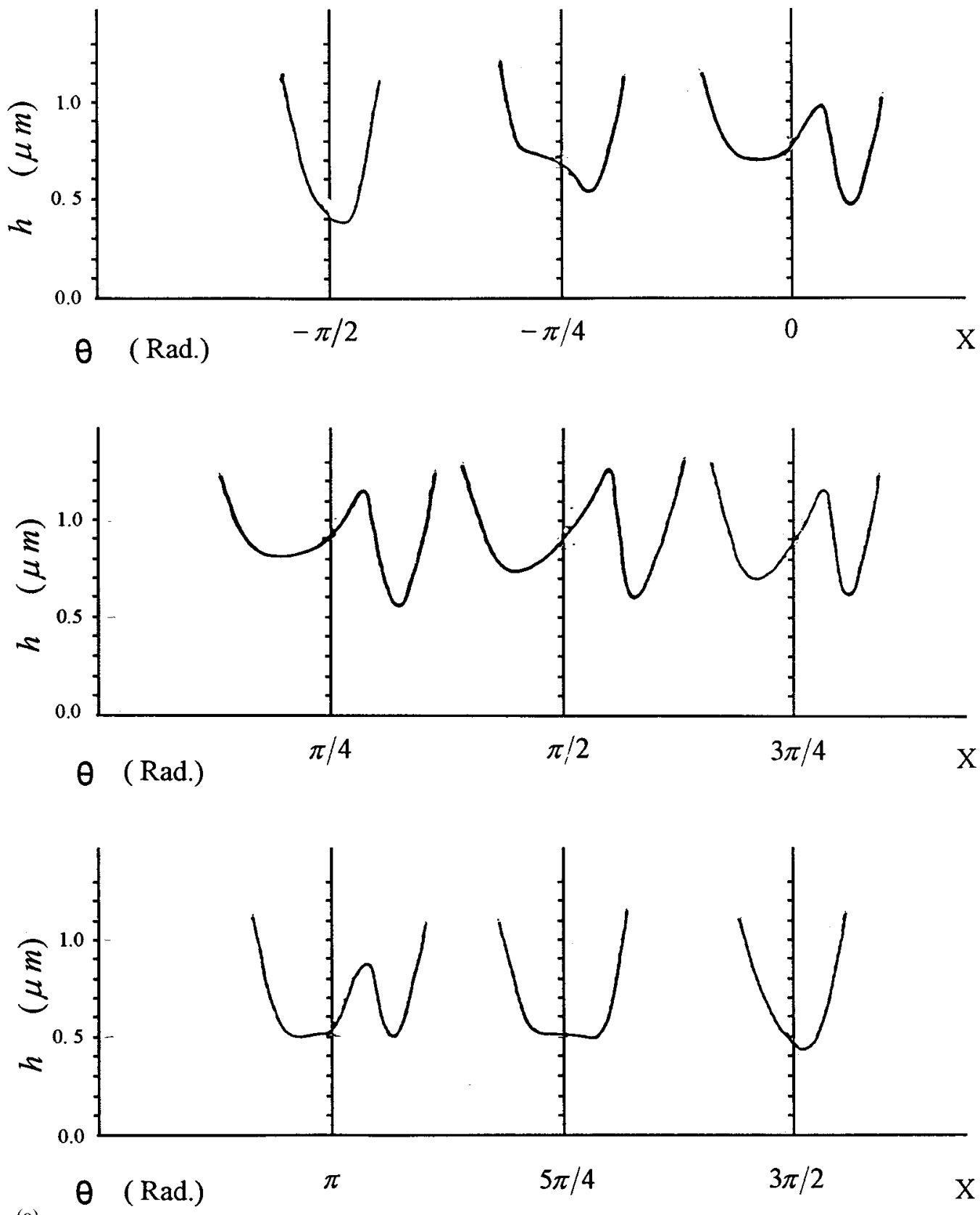

(a)

Fig. 2 (continued over) 

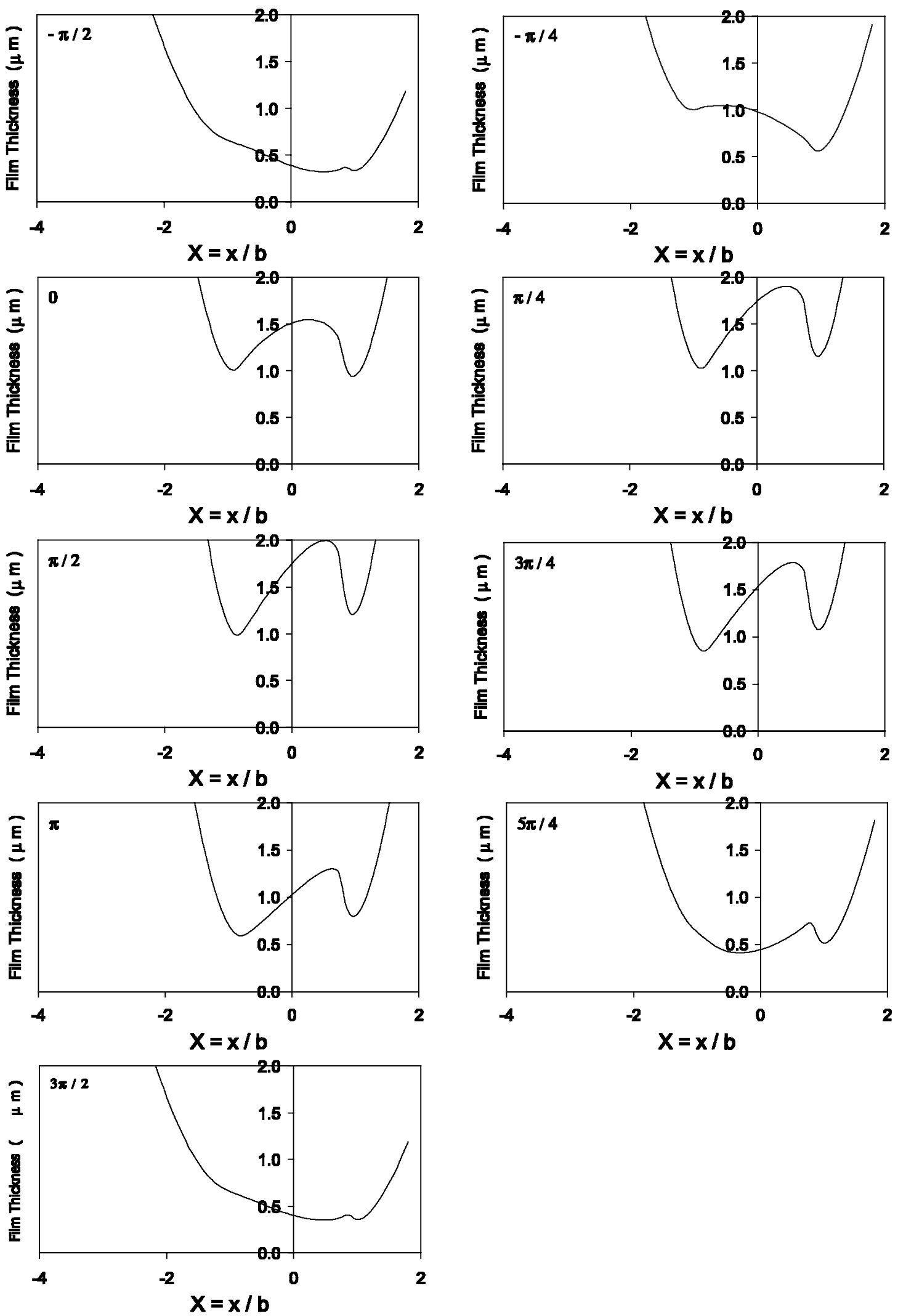

(b)

Fig. 2 (a) Experimentally measured and (b) numerically predicted transient EHL central film profiles (after Ren et al. [15]) 
of the entraining motion by as much as $0.6 \mu \mathrm{m}$. The Hertzian radius varied with the applied load during the cycle in the range $1.13 \times 10^{-4}-2.23 \times 10^{-4} \mathrm{~mm}$ in the case of numerical prediction for the same conditions as reported below.

Figure $2 \mathrm{~b}$ shows the corresponding unsteady behaviour of the EHL film, obtained here by numerical prediction. The overall conformity of the numerical results with the experimental observations of Ren et al. [15] is evident. In particular, the emergence of the dimple in the central oil-film thickness profile with an increasing value of the applied load is obtained. This central dimple is due to the increasing significance of the squeeze-film action in the $-\pi / 2, \pi / 2$ part of the load cycle. Figure 3 shows the predicted squeeze-film velocity throughout the cycle, with the numbers adjacent to the curve defining the film shapes shown in Fig. $2 b$.

The oil-film profile in Fig. $2 \mathrm{~b}$ for $\theta=0$ corresponds to the instance of maximum squeeze velocity (see point 3 in Fig. 3 and note that a negative value for squeeze velocity denotes approaching bodies). The emergence of the dimple becomes pronounced here and, owing to the dominant effect of squeeze, assumes an almost central position within the contact domain. The maximum depth for the dimple, however, occurs when the applied load reaches its peak value (i.e. at $\pi / 2$ ), and it moves towards the exit constriction on account of the effect of entraining motion when this is more dominant (see point 5 in Fig. 3). From here onwards the applied load gradually decreases and the film thickness profiles follow a similar behaviour to those obtained prior to point 5 in Fig. 3 (an almost symmetric characteristic about point 5 in Fig. 3 is observed). A quantitative comparison is shown in Fig. 4, although a close correlation between the numerical predictions and the experimental results would not be expected. This is because the conventional optical interferometric technique employed by Ren et al. [15] has been shown to be unable accurately to measure small changes in the oil-fim thickness. This argument has been corroborated by Sugimura et al. [22], who have used an interferometric method based upon spectrometer images for ultrathin films. Indeed, Ren et al. [15] have not claimed accurate film thickness measurements. Their interest has been the determination of film shape under transient conditions. To this end, a good qualitative agreement has been obtained between the numerical predictions here and their experimental findings. The apparent insensitivity of the minimum central oil-film thickness under Ren et al. [15] experiments corroborates the generally accepted EHL behaviour. However, the sensitivity of the oil-film to squeeze-film motion is also well understood $[\mathbf{3}, \mathbf{6}, \mathbf{7}, \mathbf{9}, \mathbf{1 3}]$, a fact that is not apparent from the experimental work of Ren et al. [15]. Indeed, the experimental work carried out by the same authors [15] for the finite line contact of a roller against a glass disc under transient conditions is devoid of the central dimple in the film shape with increasing load. Numerical line contact solution under non-steady state conditions show that an increasing squeeze effect results in the formation of a dimple (see, for example, reference [14]). Referring back to Fig. 4, it can be observed that good agreement is found under conditions of pure rolling and separation (at the extremities of the variations in Fig. 4). These positions correspond to

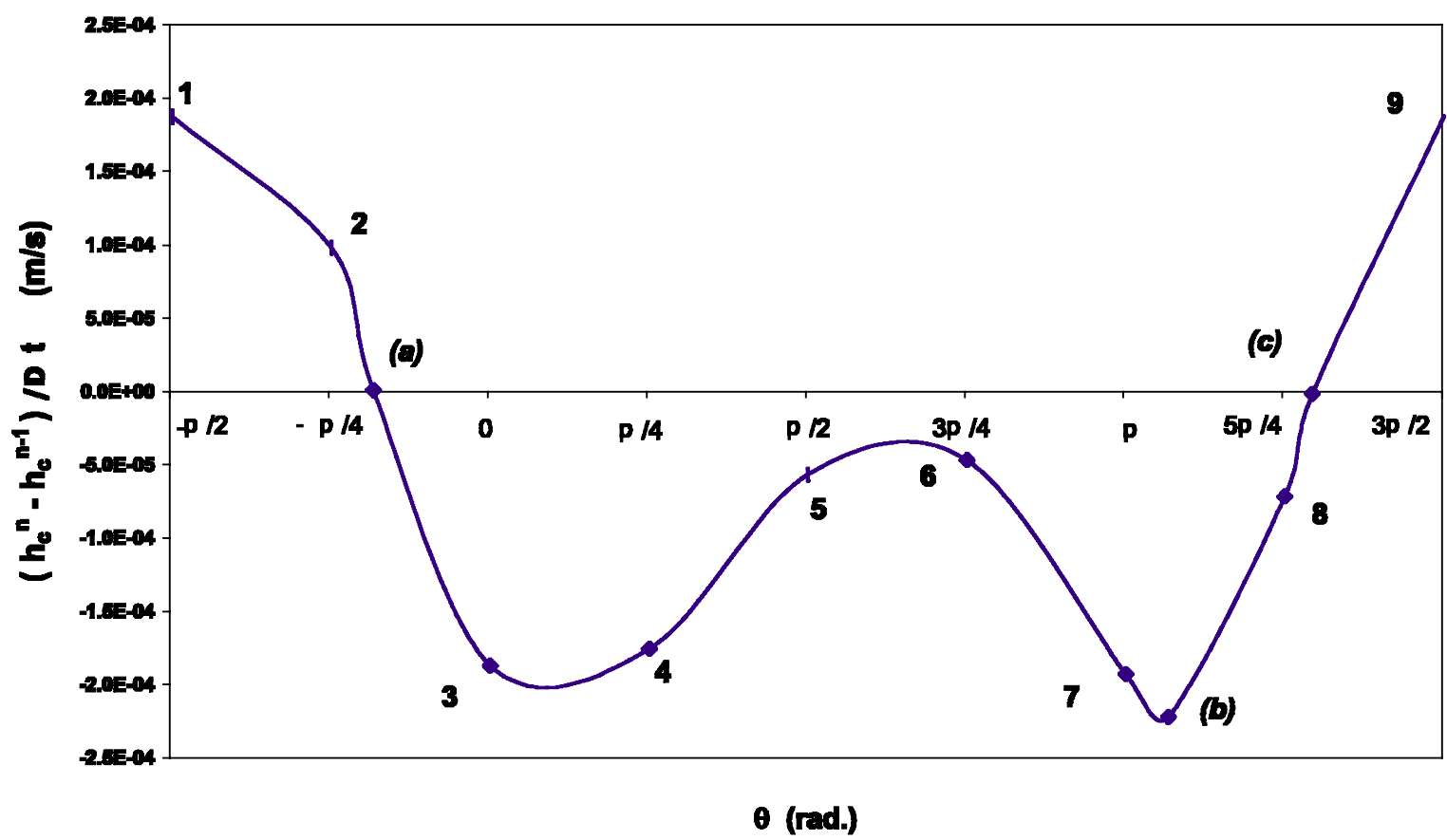

Fig. 3 Mid-contact central squeeze velocity-time history 


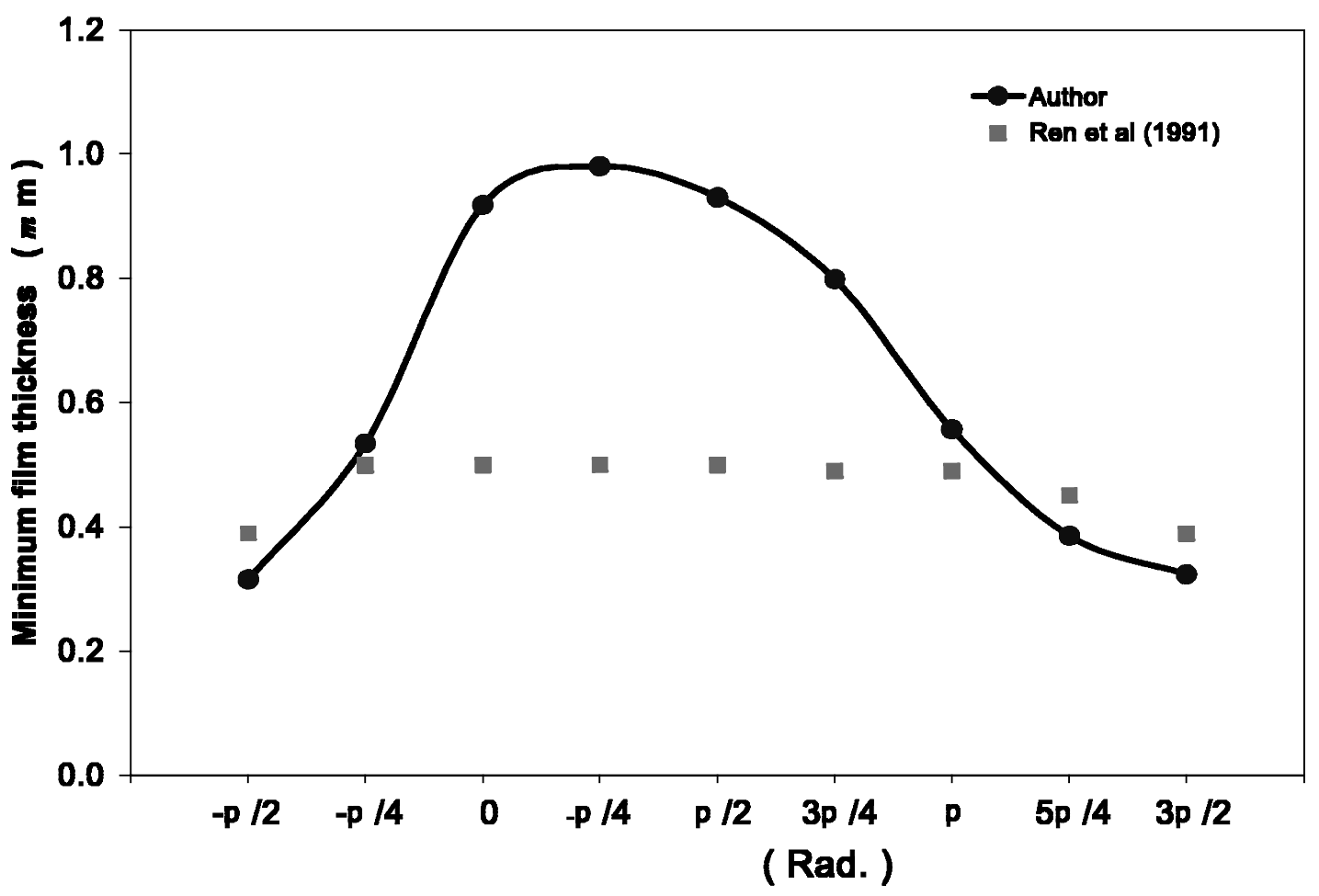

Fig. 4 Prediction of minimum film thickness variation with one cyclic load

points 1, 2, 8 and 9 in Fig. 3. A large discrepancy is noted between the numerical predictions and the experimental findings where the effect of normal approach becomes significant.

It should be noted that all numerical predictions for oscillatory point contacts exhibit the same quasiharmonic response as in the case of the numerical results presented here. The agreement between the various numerical techniques is highlighted later by a comparison between the method employed here and that reported by Oh [16], using finite element analysis.

Figure 5 shows the corresponding central pressure profiles in the direction of entraining motion for the various film thickness profiles shown in Fig. 2b. As expected, the maximum pressures occur at $\pi / 2$, where the applied load is at its maximum value. The transient pressure profiles at $\pi / 4$ and $3 \pi / 4$ are subject to the same contact load and therefore have very similar magnitudes. However, the lubricant film thickness at $\pi / 4$ (corresponding to point 4 in Fig. 3) exhibits a larger elastic indentation than that at $3 \pi / 4$ (corresponding to point 6 in Fig. 3). This is because the squeeze velocity is larger in the former, resulting in a greater film thickness at the same value of load.

To observe the effects of increasing load and the squeeze-film effect, contours of the oil-film thickness for three regions of the load cycle are shown in Fig. 6 . Figure $6 \mathrm{a}$ is the oil-film contour in the part of the cycle where the load is increasing with a load of $30 \mathrm{~N}$ and under pure entraining motion (i.e. along the time axis in Fig. 3, between points 2 and 3). Figures $6 \mathrm{~b}$ and $\mathrm{c}$ correspond to the unloading part of the cycle, with the former being at the point with the maximum squeeze velocity (i.e. between points 7 and 8 in Fig. 3). Figure $6 \mathrm{c}$ shows the oil-film contour under pure entraining motion (along the time axis in Fig. 3 between points 8 and 9). The contact loads for these cases were 30 and $10 \mathrm{~N}$ respectively. A comparison of the contours in Figs $6 \mathrm{a}$ and $\mathrm{c}$ show that the islands of the minimum oil-film thickness move from the side constrictions towards the rear exit with an increasing value of load as anticipated. The contours in Fig. 6c conform to the steady state entraining motion with the central flat region, but the contour in Fig. 6a shows the dimple that appears in the part of the cycle in which the load is increasing. Figure $6 \mathrm{~b}$ represents the same value of load as in Fig. 6a but has the maximum squeeze velocity generated during the cycle, showing a return of the minimum film thickness islands to the side constrictions. The central dimple is reduced in size as the contour corresponds to the part of the cycle in which the load is decreasing.

To obtain a verification for the numerical method presented here for the first time under non-steady conditions, a comparison is made between the present analysis and the findings by $\mathrm{Oh}$ [16], who considered a sinusoidal variation in contact load according to the relationship $W=W_{0}(1+e \sin \omega t)$, where the chosen value for $e$ 

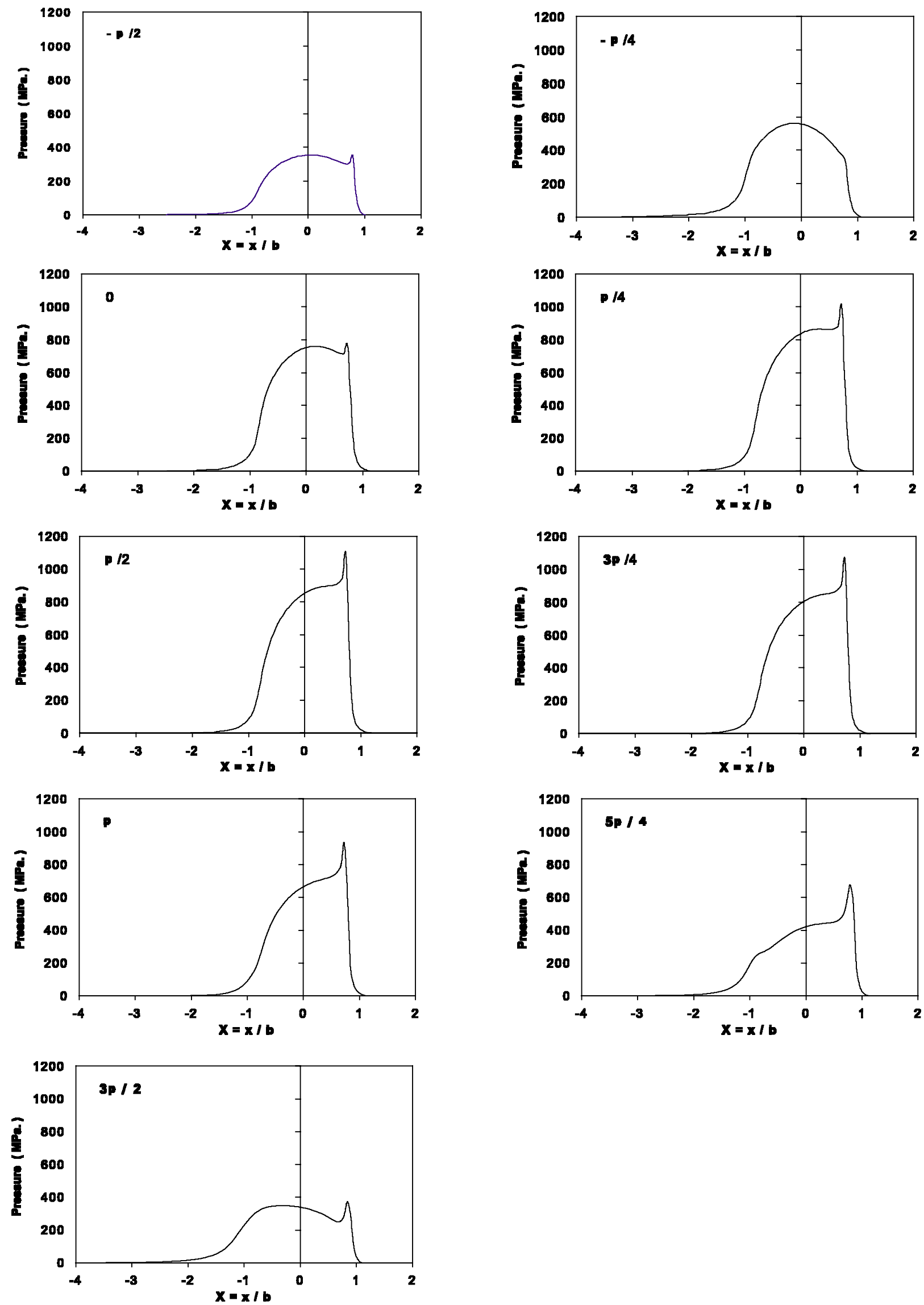

Fig. 5 Numerically predicted transient EHL central pressure profiles 

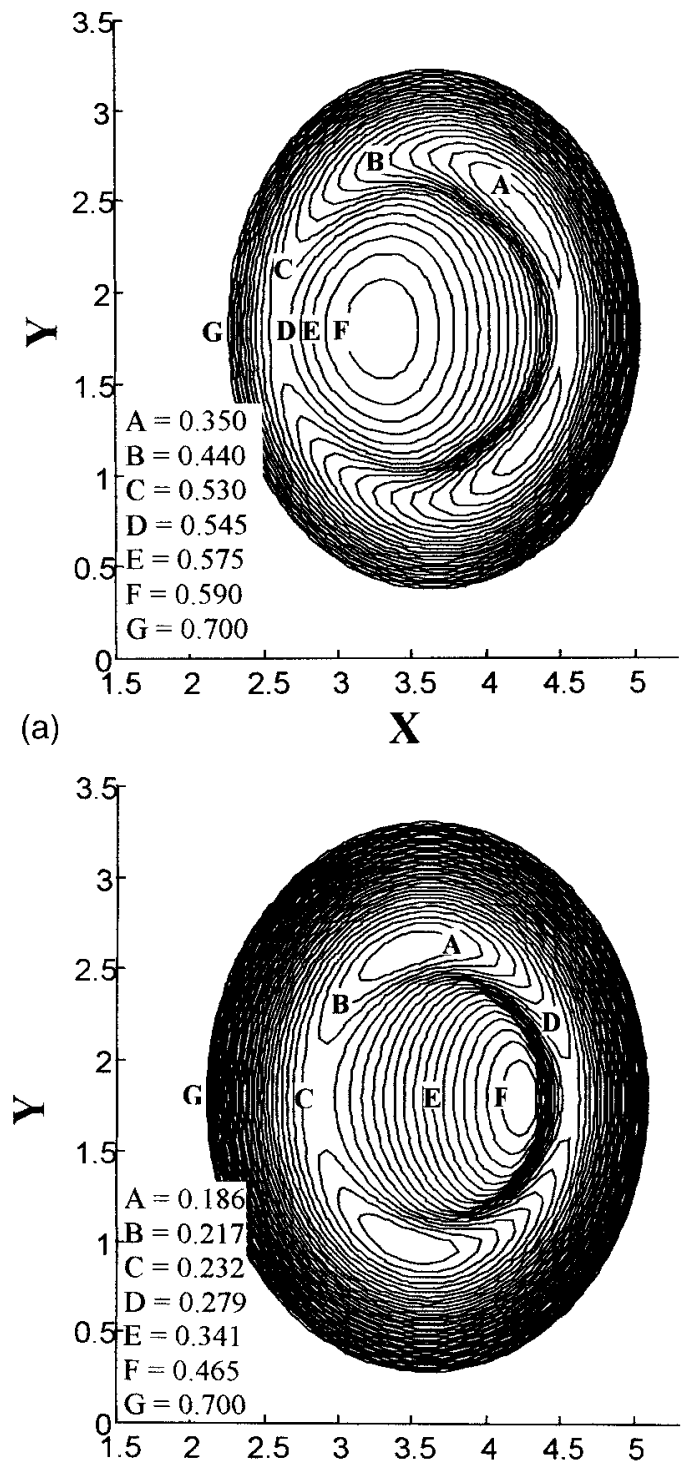

(b)

$\mathbf{X}$

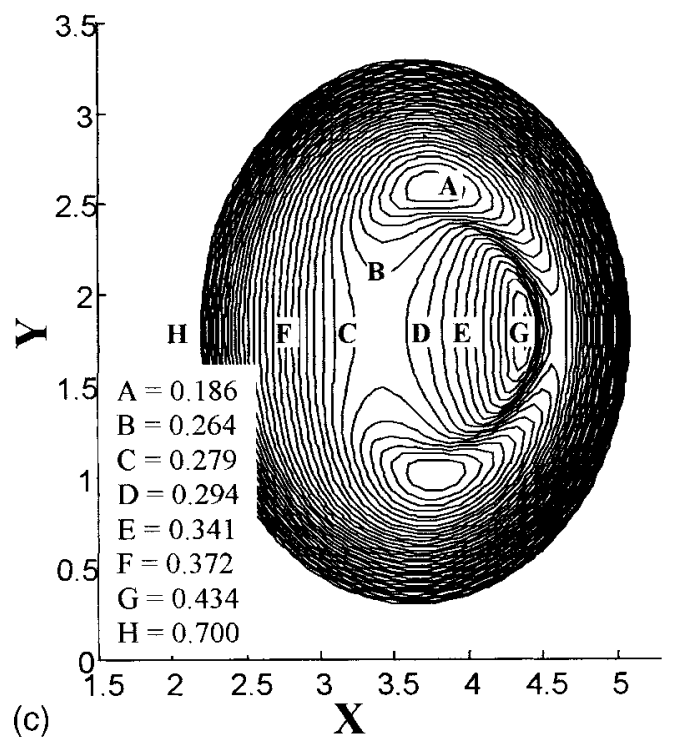

Fig. 6 Transient oil-film contour corresponding (a) to point a in Fig. 3, (b) to point b in Fig. 3 and (c) to point c in Fig. 3 
Table 2 Values of parameters used by Oh [16]

\begin{tabular}{llll}
\hline Numbers & Parameters & Symbols & Quantities and formulae \\
\hline 1 & Velocity (m/s) & $u$ & 1.98 \\
2 & Load (N) & $W$ & $W=W_{0}[1+e \sin (\omega t)]$ \\
3 & Coefficient & $e$ & 0.5 \\
4 & Nominal load (N) & $W_{0}$ & 4.15 \\
5 & Frequency (s $\left.{ }^{-1}\right)$ & $f$ & $E^{\prime} / \eta_{0}$ \\
6 & Angular velocity (s $\left.{ }^{-1}\right)$ & $\omega$ & $2 \pi f$ \\
7 & Ball radius (m) & $R$ & 0.015 \\
8 & Viscosity at $P=0(\mathrm{~Pa} \mathrm{~s})$ & $\eta_{0}$ & 0.0138 \\
9 & Poisson's ratio & $v_{1}$ & 0.3 \\
10 & Poisson's ratio & $v_{2}$ & 0.3 \\
11 & Young's modulus $(\mathrm{kPa})$ & $E_{1}$ & $96.5 \times 10^{6}$ \\
12 & Young's modulus $(\mathrm{kPa})$ & $E_{2}$ & $96.5 \times 10^{6}$ \\
\hline
\end{tabular}

was 0.5 and the frequency was $\left(\pi E^{\prime} / 2 \eta_{0}\right)$. This results in very high-frequency load oscillations with an average value of $4.15 \mathrm{~N}$.

Oh's [16] finite element results serve a useful purpose for the comparison with the finite difference approach employed in this paper. The data for the analysis are provided in Table 2. Figure 7 shows a comparison between the two sets of results. Close agreement is observed for most of the load cycle. A small divergence is observed between the two sets of results around the maximum load region of the cycle, probably owing to the relatively coarse finite element mesh employed by $\mathrm{Oh}[\mathbf{1 6}]$.

\section{CONCLUSION}

A numerical procedure that combines the NewtonRaphson scheme and low relaxation iterations has been developed for the analysis of an elastohydrodynamic circular point contact conjunction under transient isothermal conditions. The numerical results for the transient film shape conform relatively closely to the experimental findings of Ren et al. [15] and agree well

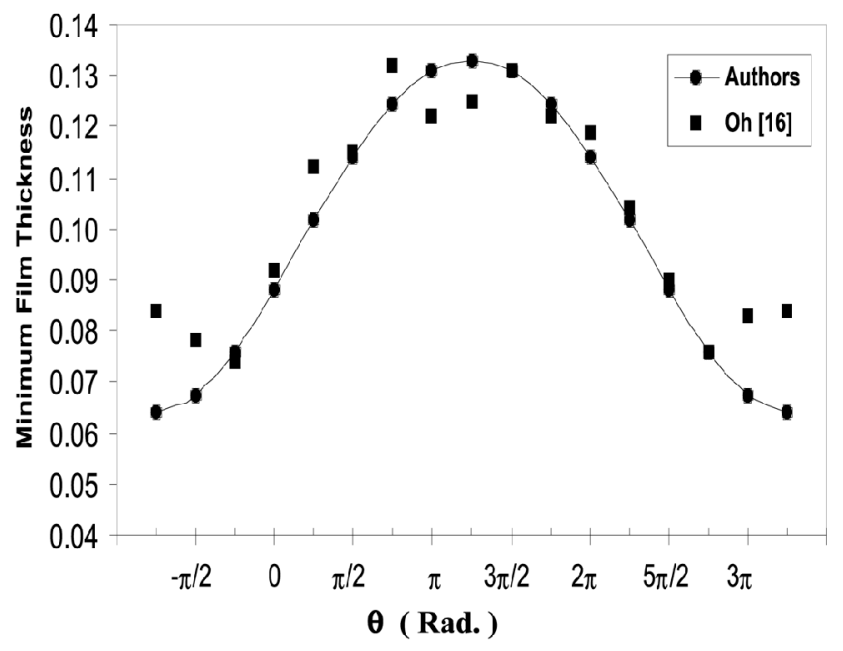

Fig. 7 Minimum film thickness variation with the experimental measurements of Nishikawa et al. [21]. Further comparisons have been made with previously reported finite element solutions for a circular point contact subjected to cyclic loading.

$\mathrm{Ai}$ and $\mathrm{Yu}[\mathbf{1 4}]$ and Ren et al. [15] have observed that the transient pressure distribution draws to the middle of the contact in that part of the cycle in which the load is increasing, with a dimple appearing in the elastic film shape. In the unloading part of the cycle, the pressure distribution spreads over the inlet and the outlet regions. The repetitive load cycles, leading to the formation of the surface dimple, can lead to premature fatigue spalling of the ball [15].

Transient conditions introduced by variation in the speed of entraining motion in a reciprocating contact show lubricant film entrapment at the ends of the stroke, where inlet reversals take place and the lubricant film is momentarily supported by pure squeeze-film action.

An important point emerging from this investigation is the dearth of experimental work for transient EHL conditions with accurate methods of film thickness measurement that correspond to conditions that are prevalent in bearings and gears subjected to cyclic loads. Recent investigations have concentrated largely upon low-load applications with ultrathin films, such as those in mechatronic machines or in instrument bearings [22].

\section{REFERENCES}

1 Christensen, H. The oil film in a closing gap. Proc. R. Soc. (Lond.) A. Math. Phys. Sci., 20 March 1962, 266(1326), 312-328

2 Herrebrugh, K., Elastohydrodynamic squeeze between two cylinders in normal approach. Trans. ASME, J. Lubric. Technol., April 1970, 92(2), 292-302.

3 Christensen, H. Elastohydrodynamic theory of spherical bodies in normal approach. Trans. ASME, J. Lubric. Technol., January 1970, 92(1), 145-164.

4 Conway, H. D. The rate of change of film thickness in the elastohydrodynamic squeeze film process. Trans. ASME, J. Lubric. Technol., 1973, 95, 391. 
5 Lee, K. M. and Cheng, H. S. The pressure and deformation profiles between two normally approaching lubricated cylinders. Trans. ASME, J. Lubric. Technol., 1973, 95, 308-317.

6 Bedewi, M. A. A., Dowson, D. and Taylor, C. M. The squeeze-film lubrication of ellipsoids. J. Phys., D: Appl. Phys., 1992, 25, A133-A140.

7 Rahnejat, H. Influence of vibration on the oil film in concentrated contacts. PhD thesis, Imperial College of Science and Technology, 1984.

8 Rahnejat, H. Computational modelling of problems in contact dynamics. J. Engng Analysis, 1985, 2(4), 192-197.

9 Mostofi, A. and Gohar, R. Oil film thickness and pressure distribution in elastohydrodynamic point contacts. Trans. ASME, J. Lubric. Technol., April 1982, 24(4), 173-182.

10 Rahnejat, H. and Gohar, R. The vibrations of radial ball bearings. Proc. Instn Mech. Engrs, Part C, Journal of Mechanical Engineering Science, 1985, 199(C3), 181-193.

11 Dowson, D. and Wang, D. An analysis of the normal bouncing of a solid elastic ball on a oily plate. Wear, 1994, 179(1-2), 29-37.

12 Safa, M. and Gohar, R. Pressure distribution under a ball impacting a thin lubricant layer. Trans. ASME, J. Lubric. Technol., 1986, 108(3), 372-376.

13 Wijnant, Y. H. and Venner, C. H. Analysis of an EHL circular contact incorporating rolling element vibration. In Proceedings of 23rd Leeds-Lyon Symposium on Tribology, September 1996, pp. 445-456 (Elsevier).
$14 \mathrm{Ai}, \mathbf{X}$. L. and Yu, H. Q. A full numerical solution for general transient elastohydrodynamic line contacts and its application. Wear, 1988, 121, 143-159.

15 Ren, N., Zhu, D. and Wen, S. Z. Experimental method for quantitative analysis of transient EHL. Tribology Int., 1991, 24(4), 225-230.

16 Oh, K.P. The numerical solution of dynamically loaded elastohydrodynamic contact as a nonlinear complementarity problem. Trans. ASME, J. Tribology, 1984, 106, 88-95.

17 Johnson, K. L. Contact Mechanics, 1985, Ch. 3, p. 54 (Cambridge University Press).

18 Dowson, D. and Higginson, G. R. A numerical solution to the elastohydrodynamic problem. J. Mech. Engng Sci., 1959, 1, 6-15.

19 Roelands, C. J. A. Correlation aspects of viscositytemperature-pressure relationship of lubricating oils. PhD thesis, Delft University of Technology, The Netherlands, 1966.

20 Hsu, C. H. and Lee, R.T. Advanced multilevel solution for elastohydrodynamic lubrication circular contact problem. Wear, 1994, 177, 117-127.

21 Nishikawa, H., Handa, K. and Kaneta, M. Behavior of EHL films in reciprocating motion. Jap. Soc. Mech. Engrs, Ser. C, 1995, 38(3), 558-567.

22 Sugimura, J., Jones Jr., W. R. and Spikes, H. A. EHD film thickness in non-steady state contacts. Trans. ASME, J. Tribology, 1998, 120, 442-452.

\section{APPENDIX 1}

\section{Contact influence matrix coefficient}

$$
\begin{aligned}
\mathbf{D}= & b\left(X+\frac{1}{2 \bar{d}}\right) \ln \left\{\frac{K\left(Y+\frac{1}{2 \bar{c}}\right)+\left[K^{2}\left(Y+\frac{1}{2 \bar{c}}\right)^{2}+\left(X+\frac{1}{2 \bar{d}}\right)^{2}\right]^{1 / 2}}{K\left(Y-\frac{1}{2 \bar{c}}\right)+\left[K^{2}\left(Y-\frac{1}{2 \bar{c}}\right)^{2}+\left(X+\frac{1}{2 \bar{d}}\right)^{2}\right]^{1 / 2}}\right\} \\
& +a\left(Y+\frac{1}{2 \bar{c}}\right) \ln \left\{\frac{\left(X+\frac{1}{2 \bar{d}}\right)+\left[K^{2}\left(Y+\frac{1}{2 \bar{c}}\right)^{2}+\left(X+\frac{1}{2 \bar{d}}\right)^{2}\right]^{1 / 2}}{\left(X-\frac{1}{2 \bar{d}}\right)+\left[K^{2}\left(Y+\frac{1}{2 \bar{c}}\right)^{2}+\left(X-\frac{1}{2 \bar{d}}\right)^{2}\right]^{1 / 2}}\right\} \\
& +b\left(X-\frac{1}{2 \bar{d}}\right) \ln \left\{\frac{K\left(Y-\frac{1}{2 \bar{c}}\right)+\left[K^{2}\left(Y-\frac{1}{2 \bar{c}}\right)^{2}+\left(X-\frac{1}{2 \bar{d}}\right)^{2}\right]^{1 / 2}}{K\left(Y+\frac{1}{2 \bar{c}}\right)+\left[K^{2}\left(Y+\frac{1}{2 \bar{c}}\right)^{2}+\left(X-\frac{1}{2 \bar{d}}\right)^{2}\right]^{1 / 2}}\right\} \\
& +a\left(Y-\frac{1}{2 \bar{c}}\right) \ln \left\{\frac{\left(X-\frac{1}{2 \bar{d}}\right)+\left[K^{2}\left(Y-\frac{1}{2 \bar{c}}\right)^{2}+\left(X-\frac{1}{2 \bar{d}}\right)^{2}\right]^{1 / 2}}{\left(X+\frac{1}{2 \bar{d}}\right)+\left[K^{2}\left(Y-\frac{1}{2 \bar{c}}\right)^{2}+\left(X+\frac{1}{2 \bar{d}}\right)^{2}\right]^{1 / 2}}\right\}
\end{aligned}
$$




\section{APPENDIX 2}

$$
\begin{aligned}
& \frac{\mathrm{d} H}{\mathrm{~d} \bar{t}}=\frac{\mathrm{d} H_{0}}{\mathrm{~d} \bar{t}}+\frac{\mathrm{d} s}{\mathrm{~d} \bar{t}}+\frac{\mathrm{d} \delta}{\mathrm{d} \bar{t}} \\
& \frac{\mathrm{d} S}{\mathrm{~d} \bar{t}}=0 \\
& \frac{\mathrm{d} H_{0}}{\mathrm{~d} \bar{t}}=\frac{H_{0}^{n}-H_{0}^{n-1}}{\Delta \bar{t}} \\
& \frac{\mathrm{d} \delta}{\mathrm{d} \bar{t}}=\frac{2}{\pi} \frac{P_{\mathrm{h}}}{E^{\prime}} \sum_{j=1,2, \ldots}^{N} \sum_{i=1,2, \ldots}^{M} D_{i^{*} j^{*}} \frac{P^{n}-P^{n-1}}{\Delta \bar{t}} \\
& \frac{\mathrm{d} H}{\mathrm{~d} t}=\frac{H_{0}^{n}-H_{0}^{n-1}}{\Delta \bar{t}}+\frac{2}{\pi} \frac{P_{\mathrm{h}}}{E^{\prime}} \sum_{j=1,2, \ldots i}^{N} \sum_{i=1,2, \ldots}^{M} D_{i^{*} j^{*}} \frac{P^{n}-P^{n-1}}{\Delta \bar{t}} \\
& \bar{\rho}_{i, j}=1+\frac{A P_{\mathrm{h}} P_{i, j}}{1+B P_{\mathrm{h}} P_{i, j}} \\
& \frac{\mathrm{d} \bar{\rho}_{i, j}}{\mathrm{~d} \bar{t}}=\frac{A P_{\mathrm{h}}}{1+B P_{\mathrm{h}} P_{i, j}}\left(1-\frac{B P_{\mathrm{h}} P_{i, j}}{1+B P_{\mathrm{h}} P_{i, j}}\right)\left(\frac{P_{i, j}^{n}-P_{i, j}^{n-1}}{\Delta \bar{t}}\right) \\
& \frac{\mathrm{d} \bar{\rho}_{i, j}}{\delta P_{i, j}}=\frac{A P_{\mathrm{h}}}{\left(1+B P_{\mathrm{h}} P_{i, j}\right)^{2}}
\end{aligned}
$$

